on current and life time measures of depression, anxiety, alcohol misuse, post-traumatic stress disorder, suicide and trauma will be presented. The findings will be discussed in the light of current policies and strategies and recommendations for further practice will be outlined.

\section{B.6 THE ECONOMIC BURDEN OF WORK-RELATED ASBESTOS EXPOSURE}

${ }^{1}$ Emile Tompa*, ${ }^{*}$ Christina Kalcevich, ${ }^{2}$ Christopher McLeod, ${ }^{3}$ Martin Lebeau, ${ }^{4}$ Chaojie Song, ${ }^{4}$ Joanne Kim, ${ }^{4}$ Paul Demers. ${ }^{1}$ Institute for Work and Health, Toronto, Canada; ${ }^{2}$ University of British Columbia, Vancouver, Canada; ${ }^{3}$ Institut de recherche Robert-Sauvé en santé et en sécurité du travail, Montreal, Canada; ${ }^{4}$ Occupational Cancer Research Centre, Toronto, Canada

\subsection{6/OEM-2019-EPI.66}

The objective of this study was to estimate the economic burden of lung cancer and mesothelioma due to occupational and paraoccupational asbestos exposure in Canada.

We estimated the lifetime cost of newly diagnosed lung cancer and mesothelioma cases associated with occupational and para-occupational asbestos exposure for calendar year 2011 based on the societal perspective. The key cost components considered were healthcare costs, productivity and output costs, and quality of life costs.

There were 427 cases of newly diagnosed mesothelioma cases and 1904 lung cancer cases attributable to asbestos exposure in our reference year-calendar year 2011-for a total of 2331 cases. Our estimate of the economic burden is \$C831 million in direct and indirect costs for newly identified cases of mesothelioma and lung cancer and \$C1.5 billion in quality of life costs based on a value of $\$ C 100,000$ per quality-adjusted life year. This amounts to \$C356,429 and \$C652,369 per case, respectively.

The economic burden of lung cancer and mesothelioma associated with occupational and para-occupational asbestos exposure is substantial. The estimate identified is for 2331 newly diagnosed, occupational and para-occupational exposure cases in 2011, so it is only a portion of the burden of existing cases in that year. Our findings provide important information for policy decision makers for priority setting, in particular the merits of banning the mining of asbestos and use of products containing asbestos in countries where they are still allowed and also the merits of asbestos removal in older buildings with asbestos insulation.

\section{Silica Exposure and Health Effects}

\section{O3C.1 SILICA EXPOSURE IN SWEDISH IRON FOUNDRIES AND BIOLOGICAL MARKERS OF INFLAMMATION AND COAGULATION IN BLOOD}

Lena Andersson*, Ing-Liss Bryngelsson, Håkan Westberg. Department of Occupational and Environmental Medicine, Örebro, Sweden

\subsection{6/OEM-2019-EPI.67}

Background Work-related exposure to silica is a health hazard worldwide causing i.e. silicosis. Inflammation is known to be a cause of cardiovascular diseases and some studies have presented elevated cardiovascular disease mortality in relation to silica exposure. The aim of this study was to investigate the relationship between inhalation of exposure to silica in Swedish iron foundries and markers of inflammation and coagulation in blood.

Methods Personal sampling of respirable dust and silica was performed for 85 subjects in three Swedish iron foundries. Stationary measurements were used to study concentrations of respirable dust and silica, inhalable and total dust, PM10 and PM2.5, the particle surface area and the particle number concentrations. The markers of inflammation analyzed were, interleukins (IL-1 $\beta$, IL-6, IL-8, IL-10 and IL-12), C-reactive protein, serum amyloid A (SAA), and markers of coagulation fibrinogen, factor VIII (FVIII), von Willebrand factor, and ddimer were measured in plasma or serum. The sampling was performed on the second or third day of a working week following a work free weekend, and additional sampling on the fourth or fifth day.

The personal and stationary measurement data were categorized into three classes to introduce high contrast with a minimum of 10 workers in each group. A mixed model analysis adjusted for sex, age, smoking, infections, blood group, sampling day and BMI was used.

Results For personal sampling the average 8 hour timeweighted average air concentration of respirable dust were $0.85 \mathrm{mg} / \mathrm{m} 3$ and respirable silica $0.052 \mathrm{mg} / \mathrm{m} 3$. For the high exposure group, statistically significant increased levels of SAA, fibrinogen and FVIII were determined for some exposure measures.

Conclusions This study may indicate an increased risk of cardiovascular disease when observing relations between particle exposure and inflammatory markers.

\section{O3C.2 A 30-YEAR IMPACT ANALYSIS OF BEST PRACTICES FOR SILICA DUST EXPOSURE REDUCTION IN CONSTRUCTION: COSTS, BENEFITS AND HRQL}

${ }^{1}$ Emile Tompa*, ${ }^{1}$ Amirabbas Mofidi, ${ }^{3}$ Young Jung, ${ }^{2}$ Chaojie Song, ${ }^{2}$ Kate Jardine, ${ }^{2}$ Victoria Arrandale, ${ }^{4}$ Thomas Tenkate, ${ }^{5}$ Hugh Davies, ${ }^{2}$ Paul Demers. ${ }^{1}$ Institute For Work and Health, Toronto, Canada; ${ }^{2}$ Occupational Cancer Research Centre, Toronto, Canada; ${ }^{3}$ McMaster University, Hamilton, Canada; ${ }^{4}$ Ryerson University, Toronto, Canada; ${ }^{5}$ University of British Columbia, Vancouver, Canada

\subsection{6/OEM-2019-EPI.68}

Crystalline silica dust exposure is common in the construction sector. In fact, it is the industry with the largest number of people exposed in Canada. Inhalation of silica dust is known to cause lung cancer, silicosis, chronic obstructive pulmonary diseases, rheumatoid arthritis, and tuberculosis. The hierarchy of controls provide a range of prevention options for reducing or eliminating exposure. In this study we undertake an impact analysis of the implementation of two exposure reduction approaches-use of personal protective equipment by all exposed individuals and use of engineering controls wherever and whenever feasible.

We estimated the intervention costs over a 30 year time period (2020-2050) of each exposure reduction approach implemented across the entire sector in Ontario, Canada, and considered equipment, maintenance, training, and productivity costs of each approach over the time period. The economic impacts from lung cancer cases averted due to exposure reduction included three broad categories of impacts-direct, indirect and intangible. We took a societal- 